\title{
Estudos Empíricos Aplicados ao Management Function Deployment (MFD)
}

\begin{tabular}{|c|c|c|c|c|}
\hline $\begin{array}{l}\text { Cunha Júnior, H. B. } \\
\text { Escola Politécnica de Per- } \\
\text { nambuco } \\
\text { Universidade de Pernam- } \\
\text { buco } \\
50.720-001 \text { - Recife, Bra- } \\
\text { sil } \\
\text { hbjc@ecomp.poli.br }\end{array}$ & $\begin{array}{l}\text { Coutinho, L. } \\
\text { D. } \\
\text { Escola Politéc- } \\
\text { nica de Pernam- } \\
\text { buco } \\
\text { Universidade de } \\
\text { Pernambuco } \\
50.720-001 \text { - } \\
\text { Recife, Brasil } \\
\text { ldc@ecomp.pol } \\
\text { i.br }\end{array}$ & $\begin{array}{l}\text { Aguiar, B. C. G. } \\
\text { Escola Politécnica } \\
\text { de Pernambuco } \\
\text { Universidade de } \\
\text { Pernambuco } \\
50.720-001 \text { - Re- } \\
\text { cife, Brasil } \\
\text { bcga@ecomp.poli. } \\
\text { br }\end{array}$ & $\begin{array}{l}\text { Silveira, D. S. } \\
\text { Escola Politécnica } \\
\text { de Pernambuco } \\
\text { Universidade de } \\
\text { Pernambuco } \\
\text { 50.720-001 - Re- } \\
\text { cife, Brasil } \\
\text { de- } \\
\text { nis@ecomp.poli.br }\end{array}$ & $\begin{array}{l}\text { Carvalho, G. H. P. } \\
\text { Escola Politécnica de } \\
\text { Pernambuco } \\
\text { Universidade de Per- } \\
\text { nambuco } \\
\text { 50.720-001 - Recife, } \\
\text { Brasil } \\
\text { gustavohpcarva- } \\
\text { lho@ecomp.poli.br }\end{array}$ \\
\hline
\end{tabular}

Resumo Aumentar a maturidade dos processos de gerenciamento dos projetos é uma preocupação enfatizada por vários modelos de maturidade de processos existentes. Porém, deseja-se também aumentar a importância estratégica da melhoria destes processos. O método Management Function Deployment (MFD), inspirado no Quality Function Deployment (QFD), foi proposto justamente com o intuito de viabilizar este alinhamento. Portanto, este artigo analisa as técnicas de alinhamento estratégico da melhoria de processos, a partir de uma revisão sistemática da liretura, como também propõe uma ferramenta de apoio ao uso de técnicas baseadas no QFD.

\footnotetext{
Abs- $\quad$ Increasing the maturity of project management processes is one concern of many process maturity motract dels. Besides increasing, it is also important to pay attention to the strategic importance of these processes improvements. The Management Function Deployment (MFD) method, an adaptation of the Quality Function Deployment (QFD), has been proposed with the purpose of make this alignment feasible. Therefore,

this article analyses the techniques for strategic alignment of process improvements based on a systematic literature review and also proposes a tool to support the use of techniques inspired on QFD.
} 


\section{Introdução}

Aumentar a maturidade dos processos de gerenciamento dos projetos é uma preocupação enfatizada pelos vários modelos existentes: o Organizational Project Management Maturity Model (PMI, 2008); o Project Management Maturity Model (CRAWFORD, 2006); o Portfolio, Programme and Project Management Maturity Model (OGC, 2008); o PRINCE2 Maturity Model (OGC, 2006); o Kerzner Project Management Maturity Model (KERZNER, 2005) e o Modelo de Maturidade em Gerenciamento de Projetos (PRADO, 2008).

Porém, deseja-se também aumentar a importância estratégica da melhoria destes processos. Este desejo baseiase na premissa de que alinhar a melhoria das práticas de gerenciamento de projetos às estratégias do negócio facilita significativamente o alcance das estratégias organizacionais (SRIVANNABOON, 2006). No entanto, apesar de alguns modelos já terem incorporado a preocupação com o alinhamentoestratégico, ainda são poucos os estudos que oferecem guias práticos de como alcançar este alinhamento.

Para suprir esta lacuna, propôs um método, denominado Management Function Deployment (MFD) (CARVALHO; SILVA; FRANÇA, 2010), o qual se baseia no método Quality Function Deployment (QFD) (COHEN, 1995). Através do MFD, é possível identificar quais melhorias nos processos de gerenciamento de projetos devem ser priorizadas em função dos objetivos estratégicos traçados pela organização.

Mais recentemente, uma primeira adaptação do MFD foi criada com o intuito de permitir o alinhamento estratégico da melhoria dos processos de incubação presentes no Centro de Referência para Apoio a Novos Empreendimentos: o Incubation Function Deployment (IFD) (CARVALHO; SILVA, 2010).

Apesar destas iniciativas, é preciso realizar uma revisão da literatura mais aprofundada com o intuito de verificar que outras alternativas a literatura apresenta para o alinhamento estratégico da melhoria de processos. Além disso, é também importante levantar que ferramentas existem para apoiar o uso de técnicas baseadas no QFD.

\section{Problema de Pesquisa e Objetivos}

Portanto, este projeto está associado à investigação dosseguintes problemas de pesquisa:

- $\quad[\mathrm{P} 1]$ Que técnicas existem para alinhar estrategicamente a melhoria de processos?
- $\quad$ [P2] Quais ferramentas existem para apoiar o uso de técnicas baseadas no QFD?

A partir destes problemas são traçados os objetivos principais desta pesquisa:

- [P1-O1] Elaborar o protocolo de uma revisão sistemática da literatura capaz de responder o primeiro problema desta pesquisa $(\mathrm{P} 1)$.

- [P1-O2] Realizar uma revisão sistemática aderente ao protocolo definido por P1-O1.

- [P1-O3] Realizar uma análise quantitativa e qualitativa a partir dos trabalhos catalogados e analisados na revisão sistemática da literatura.

- [P2-O1] Levantar os requisitos funcionais e nãofuncionais de uma ferramenta computacional para auxiliar o planejamento e a aplicação do método QFD e suas variações, em particular, o MFD e o IFD.

- [P2-O2] Projetar uma arquitetura com baixo acoplamento, alta coesão e extensível para a ferramenta computacional descrita em O1.

- [P2-O3] Desenvolver um software portável que satisfaça os requisitos funcionais e não funcionais associados à $\mathrm{P} 2-\mathrm{O} 1$ e que seja aderente à arquitetura fruto de $\mathrm{P} 2-\mathrm{O} 2$.

\section{Metodologia}

O desenvolvimento da pesquisa será baseado no: (i) planejamento e execução de um estudo empírico secundário, uma revisão sistemática da literatura e (ii) planejamento e desenvolvimento de um software. Desta forma, pretende-se alcançar os objetivos listados anteriormente. Para tanto, foram delineadas as seguintes etapas:

- [E1] Nivelar o conhecimento dos participantes em relação aos conceitos-chave: (a) modelos de maturidade, (b) modelos de referência de processos, (c) modelos de avaliação de processos, (d) gerenciamento de projetos, (e) planejamento estratégico, (f) modelos de maturidade em gerenciamento de projetos e ( $\mathrm{g}$ ) alinhamento estratégico.

- [E2] Revisar a literatura para identificar e analisar trabalhos recentes relacionados a métodos que permitam o alinhamento estratégico da melhoria de processos, em particular, métodos que sejam derivados do QFD. 
- [E3] Definir os requisitos previstos em P2-O1.

- [E4] Projetar a arquitetura prevista em P2-O2.

- [E5] Desenvolver a ferramenta computacional prevista em P2-O3.

- [E6] Escrever artigo(s) e relatório(s) técnico(s).

\section{Metodologia}

Os primeiros materiais utilizados nesta pesquisa constituíram-se de artigos cedidos tanto pelo professor responsável como encontrados na internet, além da dissertação de mestrado (CARVALHO, 2010), onde foram abordados os temas pertinentes ao projeto. A partir das leituras realizadas, foi possível aprender e entender os conceitos básicos referentes ao assunto em questão. Dentre eles, pode-se explicitar:

- Melhoria dos processos de gerenciamento de projetos de software: ações que têm o objetivo de melhorar a capacidade dos processos de gerenciamento.

- Alinhamento estratégico: garantir que a melhoria dos processos de gerenciamento sejam guiados pelas metas da instituição, e não apenas pelas práticas da engenharia de software.

- Modelos de maturidade: permitem avaliar a qualidade dos processos de desenvolvimento e gerenciamento de acordo com normas e modelos definidos na literatura.

Além dos conceitos citados acima, foram estudadas as definições dos métodos Quality Function Deployment (QFD) e Management Function Deployment (MFD).

- QFD: é um método para o planejamento estruturado de produtos que explicita as necessidades do cliente podendo assim analisar as possíveis soluções propostas e seus respectivos impactos na satisfação dessas necessidades.

- MFD: é um método para o planejamento estruturado das melhorias dos processos de gerenciamento de software, avaliando cada melhoria quanto à contribuição para a satisfação das metas estratégicas. A Figura 1 apresenta algumas das matrizes do MFD, especificamente as constituintes da House of Management (HoM), cujo preenchimento guia o uso deste método.

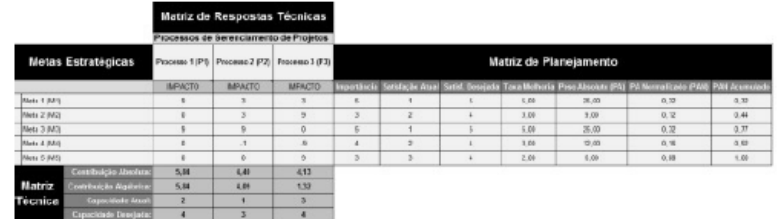

Figura 1. Matrizes da House of Management (HoM).

De forma sucinta, a HoM permite identificar quais processos de gerenciamento (listados na Matriz de Respostas Técnicas) são mais importantes em função das metas estratégicas (listadas em Metas Estratégicas) mais importantes. A identificação do que virá a ser mais importante dáse pelo preenchimento e cálculos realizados nas matrizes de Planejamento (prioriza as metas) e Técnica (prioriza os processos em função das metas priorizadas).

A partir da HoM é construída um outro conjunto de matrizes. denominadas House of Action (HoA) que tem como entrada algumas das matrizes preenchidas e calculadas da HoM. De forma análoga, a HoA permite priorizar ações de melhoria de processos em função dos processos priorizados na HoM.

Finalizado esse conjunto de leitura, deu-se início ao estudo de protocolos de Revisão Sistemática de Literatura (RSL). Foi feita uma RSL sobre alinhamento estratégico da melhoria de processos para identificar os trabalhos recentes, além dos já conhecidos, relacionados ao tema. De acordo com Kitchenham (2007), uma RSL diferencia-se de uma revisão bibliográfica por fazer esta de forma sistemática com uma grande preocupação de não introduzir viés nos resultados. Para tanto, Kitchenham sugere que uma RSL seja estruturada conforme as seguintes fases:

- Planejamento: necessário para confirmar a necessidade de uma RSL. É nessa fase que o protocolo formal de pesquisa, de acordo com um conjunto de regras, é elaborado. O protocolo é uma descrição sistemática e completa de como a pesquisa será feita.

- Condução: com o protocolo de pesquisa feito, é determinada uma estratégia de pesquisa e a revisão poderá ser iniciada. Em seguida, os trabalhos selecionados pela pesquisa são lidos e analisados.

- Disseminação: finalizada a análise dos artigos, os resultados potencialmente relevantes serão listados e disseminados na comunidade associada.

Foi estudado também os conceitos de modelagem de software. O uso efetivo desta prática da engenharia de software se faz necessário para que exista um bom planejamento do desenvolvimento de um software, antes do início 
do desenvolvimento do mesmo. Esta ação antecipa problemas e, faz com que o desenvolvimento do programa seja mais fácil, menos susceptível a problemas, além de promover o reuso do código. Portanto, foi necessário um estudo sobre esses conceitos, especificamente a elaboração de casos de uso e suas definições. como também dos diagramas de classe conceituais. Para tanto, fez-se uso de livros da biblioteca e materiais disponíveis na internet.

Além do estudo dos tópicos acima listados, fez-se o estudo, através de livros e fontes na internet, da linguagem de programação Action Script.

\section{Resultados Finais}

Os resultados finais constituem-se das conclusões da Revisão Sistemática da Literatura, associados aos objetivos [P1-O1, P1-O3], e da arquitetura e protótipo de uma ferramenta de apoio ao QFD e MFD, associado aos objetivos [P2-O1, P2-O3].

\subsection{Protocolo e Resultados da Revisão Siste- mática da Literatura}

A realização deste estudo secundário da Engenharia de Software experimental se deu com a finalidade de pesquisar por trabalhos científicos relacionados ao problema 1 (P1). Dessa forma, se faz necessário a elaboração de um protocolo de pesquisa, pois essa é uma ferramenta sistemática, ou seja, caso outra pessoa deseje refazer essa mesma pesquisa, seguindo o passo a passo do protocolo, ela irá obter, no mínimo, os mesmos resultados.

Primeiramente, foram definidas duas questões de pesquisa que iriam englobar todo o contexto deste projeto. Foram elas:

- Q1: Como alinhar a melhoria de processos de software, descritos nos modelos de maturidade, às estratégias de negócio?

- Q2: Como utilizar o QFD na melhoria de processos de software descritos nos modelos de maturidade?

Feito isso, definiu-se que a base de busca dos artigos científicos seria o site do IEEEXplore Digital Library. Outras bases de artigos não foram consideradas por limitação de tempo. Foram definidas as palavras-chave para cada questão de pesquisa, e, posteriormente, traduzidas para a língua inglesa. O próximo passo foi a análise de como essas palavras deveriam ser organizadas para que fossem retornados os artigos relevantes ao tema em questão. Para tanto, fez-se uso da construção de uma forma normal conjuntiva.

Assim, foram formadas as duas strings de busca, uma para cada questão de pesquisa, e elas foram utilizadas para iniciar a busca no site citado acima. São elas:

- $\quad$ S1: ((()((process *) AND improve*) AND ((strateg*) OR business)) AND align*) AND software) AND "maturity model*"

- $\quad S 2$ : ((()(process*) AND improve*) AND ((QFD) OR"quality function deployment")) AND software) AND "maturity model ${ }^{* \prime)}$

Baseados em critérios de inclusão e exclusão de que artigos seriam lidos e quais não seriam, assim como em um procedimento de aplicação destes critérios, dos 57 artigos retornados para a primeira string e dos 16 artigos retornados para a segunda string, 15 e 9 foram selecionados respectivamente, totalizando 24 trabalhos.

Em seguida, cada um destes 24 artigos foi lido e alguns dados foram extraídos dos mesmos, conforme o formulário de coleta de dados apresentado na Tabela 2 . O objetivo deste formulário é extrair as principais informações de cada um dos artigos, para, posteriormente, ser feita uma análise quantitativa e qualitativa dos artigos lidos.

Tabela 2. Formulário de Coleta de Dados da RSL.

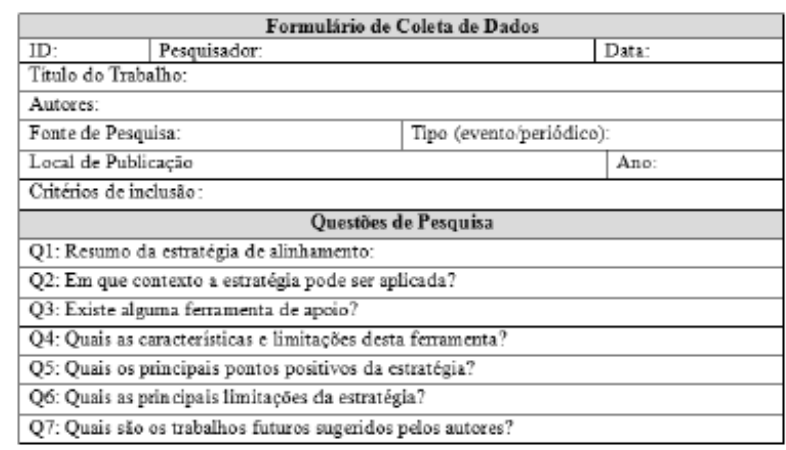

Em seguida, com o intuito de avaliar o quanto estes trabalhos contribuem para responder o problema de pesquisa $\mathrm{P} 1$, elaborou-se um formulário de avaliação da qualidade (Tabela 3) que foi preenchido para cada estudo lido. A coluna valores foi respondida de acordo com a seguinte escala:

- Discordo plenamente: 1 ponto

- Discordo: 2 pontos;

- $\quad$ Neutro: 3 pontos; 
- Concordo: 4 pontos;

- Concordo plenamente: 5 pontos.

Tabela 3. Formulário de Avaliacão da Qualidade.

\begin{tabular}{|c|l|c|}
\hline \multicolumn{3}{|c|}{ Avaliação de Qualidade } \\
\hline Item & \multicolumn{1}{|c|}{ Critérios de Qualidade } & Valores \\
\hline $\mathbf{1}$ & As perguntas Q2 a Q7 foram respondidas corn exatidão? & \\
\hline $\mathbf{2}$ & O artigo descreve o processo metodológico de fomma replicável? & \\
\hline $\mathbf{3}$ & As respostas das perguntas Q2 e Q5 são baseadas em evidências? & \\
\hline
\end{tabular}

Em seguida, para calcular a qualidade de um trabalho, calculou-se o percentual de pontos obtidos em relação ao máximo possível ( 3 questões $\mathrm{x} 5$ pontos $=15$ pontos) $\mathrm{e}$ utilizaram-se os estratos propostos por Beecham et al. (2007) que podem ser vistos na Tabela 4.

Tabela 4. Estratos de Qualidade.

\begin{tabular}{|c|c|}
\hline Faixa de Notas & Estratos \\
\hline$>86 \%$ & Excelente \\
\hline $66 \% \leq<85 \%$ & Muito Boa \\
\hline $46 \% \leq<65 \%$ & Boa \\
\hline $26 \% \leq<45 \%$ & Media \\
\hline$<26 \%$ & Baixa \\
\hline
\end{tabular}

Finalizado todo o processo de busca e leitura dos artigos relevantes, a análise dos mesmos foi realizada. Primeiramente, fez-se uma análise quantitativa e em seguida uma qualitativa. Os gráficos a seguir apresentam uma visão geral dos resultados da análise quantitativa.

\section{Artigos por Tipo de Publicação}

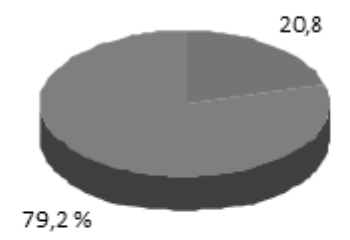

- Periódicos

Anais

Gráfico 1. Distribuição por Tipo de Publicação.

O Gráfico 1 mostra que a maioria dos trabalhos ainda são resultados preliminares uma vez que a grande maioria dos mesmos foram publicados em eventos, enquanto que a minoria em periódicos.

\section{Artigos por Ano de Publicação}

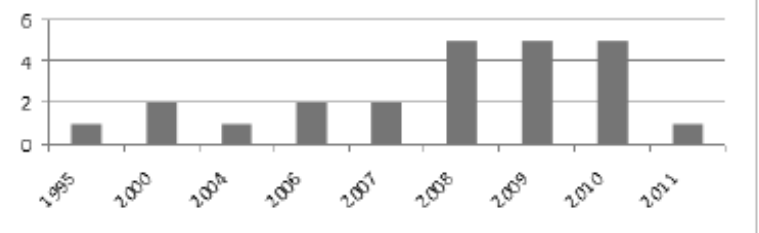

Gráfico 2. Distribuição por Ano de Publicação.

Já o Gráfico 2 mostra que esta área de estudo é relativamente recente, o artigo mais antigo é de 1995, e que o interesse pela mesma tem crescido nos últimos anos como mostra a maior quantidade de trabalhos publicados nos últimos anos.

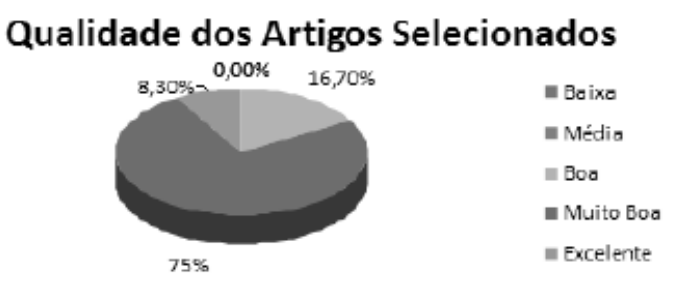

Gráfico 3. Distribuição da Qualidade dos Artigos Selecionados.

Como pode-se notar no Gráfico 3, a maioria dos artigos lidos teve uma classificação muito boa e nenhum com uma qualidade baixa ou regular. O que mostra que as strings de busca conseguiram capturar artigos relevantes que ajudam a resposta a pergunta de pesquisa $\mathrm{P} 1$ do estudo.

Quanto à análise qualitativa, ao ser realizada a leitura dos artigos inclusos relacionados à string 1, foi possível perceber que uma grande quantidade deles possuía como foco principal ressaltar para as empresas, no ambiente competitivo de negócios atual, a importância do alinhamento estratégico de tais negócios com a melhoria dos processos de software.

Esses artigos citavam como o planejamento estratégico é uma das etapas chaves para uma melhor concretização dos objetivos da empresa, como deve estar relacionado a estratégias de marketing competitivas e que alinhamento pode ser visto como aplicação de TI de forma adequada e oportuna, em harmonia com as estratégias de negócio, com os objetivos e necessidades. Vários deles enfatizam a necessidade do treinamento e educação dos profissionais de TI na área de negócios e liderança para alcançar os objetivos do alinhamento estratégico. 
Em um dos artigos selecionados, foi analisado o uso de técnicas de desenvolvimento ágil em conjunto com alinhamento estratégico e concluiu-se que, embora nem sempre essas técnicas ágeis sejam eficientes, quando utilizadas com o alinhamento, melhoram seu desempenho. Os artigos relatam também as vantagens dessa prática para as empresas e apresentam, em níveis variados de profundidade e detalhamento, o passo a passo de como iniciar e pôr em prática dentro das empresas essa idéia.

Quanto aos artigos inclusos ligados à string 2, foi encontrado desde a simples explicação do que é o QFD até diversas aplicações dessa técnica, ou sua combinação com algumas outras.

Em um deles, a fim de obter as necessidades do cliente da forma mais eficiente possível, foi combinado com o QFD o uso de outra técnica, conhecida com lógica fuzzy. A razão da escolha dessa nova técnica deve-se ao fato da pesquisa estar inserida em um contexto repleto de variáveis incertas, e fuzzy é ideal quando se torna necessário lidar com ambientes nebulosos.

Em outro, citou-se um método semelhante ao QFD chamado SERVQUAL, desenvolvido em 1988. Era um software piloto, mas detectou-se que vários estudos sobre a teoria desse novo método necessitavam ser realizados.

Vale ressaltar que foi encontrado um artigo que fez uso de uma das ferramentas pagas pesquisadas no início deste projeto de pesquisa chamada QFDCapture. O contexto do artigo estava inserido no Ensino Superior e tinha como objetivo aumentar a qualidade da educação nas Universidades e Escolas chinesas, local onde foi realizada a pesquisa.

Portanto, observou-se que as técnicas de alinhamento propostas estão sendo cada vez mais utilizadas, visto que elas priorizam as necessidades de uma empresa. Contudo, mas que ainda é um campo de pesquisa em aberto.

\subsection{Modelagem e Desenvolvimento de Ferra- mentas de Apoio ao QFD e MFD}

Como um dos objetivos desta pesquisa é desenvolver uma ferramenta que auxilie o uso de técnicas baseadas no $\mathrm{QFD}$, inicialmente, fez-se uma pesquisa na internet de ferramentas de apoio ao uso do QFD. Como apresentado na Tabela 5, existem algumas ferramentas, mas nenhuma delas é gratuita. Com base nas funcionalidades presentes nestas ferramentas, como também em funcionalidades ausentes, mas desejadas, realizou-se um levantamento dos requisitos que deveriam ser implementados no software.
Tabela 5. Comparação de Ferramentas de Apoio ao QFD.

\begin{tabular}{|l|c|c|}
\hline \multicolumn{1}{|c|}{ Ferramenta } & Idioma & Gratuita \\
\hline Path Maker & Inglês & Não \\
\hline Product Development Toolkit & Inglês & Não \\
\hline QFDcapture Professional Edition & Inglês & Não \\
\hline Logic MindGuide & Alemão & Não \\
\hline Qualica QFD & Inglês e Alemāo & Não \\
\hline QFD Designer V4 & Inglês & Não \\
\hline CIMOS QFD 2.0 & Inglês & Não \\
\hline
\end{tabular}

Feito isso, foi elaborado um Diagrama de Casos de Uso que auxilia o desenvolvedor a construir um cenário representando as funcionalidades do sistema do ponto de vista do usuário. Para representar esse diagrama, foi utilizada a Unified Modeling Language (UML) já que ela apresenta uma notação gráfica. Para cada caso de uso, definiu-se também as pré e póscondições dos mesmos. A Figura 2 apresenta os casos de uso identificados.

Finalizada a construção do diagrama de casos de uso, o próximo passo foi elaborar o Diagrama de Classes. Diferente do primeiro, esse diagrama é direcionado para o desenvolvimento do software, visto que ele é uma representação da estrutura e relações das classes do programa. Neste diagrama devem estar contidas todas as classes que pertençam ao código-fonte da ferramenta. As Figuras 3, 4 e 5 apresentam os principais diagramas de classes da ferramenta. Como prática de modularidade, a arquitetura da ferramenta baseou-se no padrão MVC (Model View Control) no qual são separadas em diferentes camadas: (i) as classes que manipulam a interface gráfica (View), (ii) as classes básicas do sistema (Model) e (iii) as classes que controlam o uso das classes básicas (Control) a partir das requisições feitas pela interface gráfica.

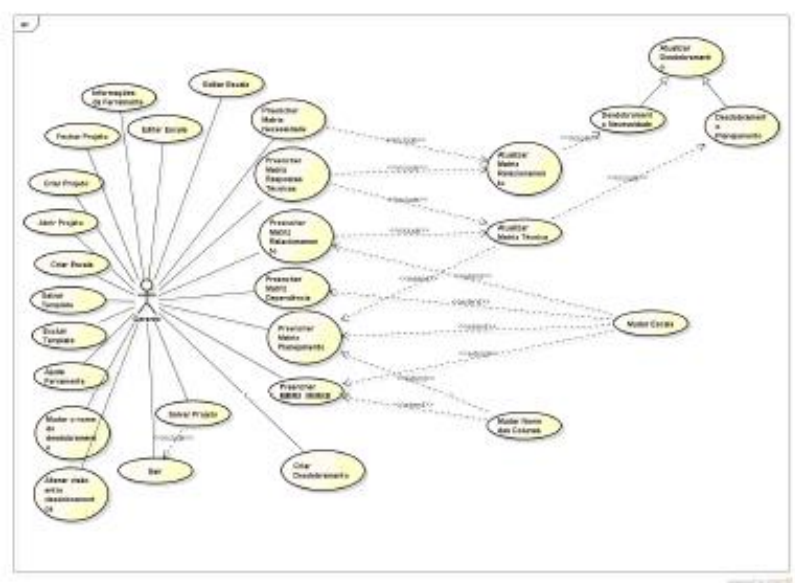

Figura 2. Casos de Uso da Ferramenta. 


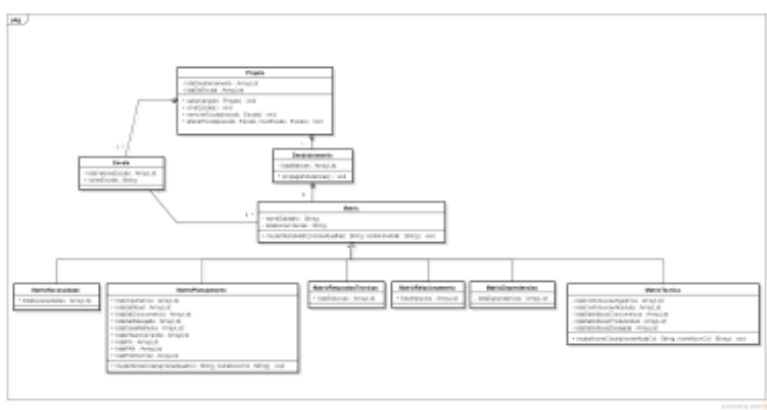

Figura 3. Classes Básicas da Ferramenta.

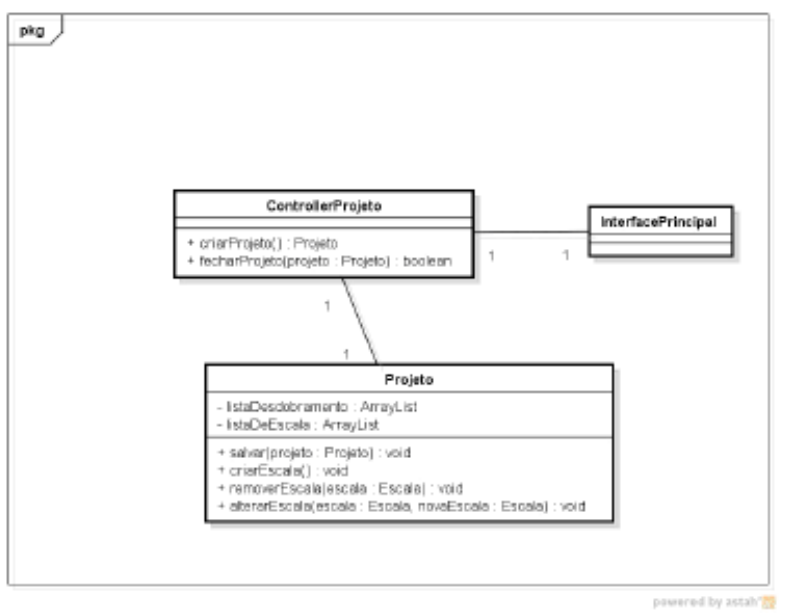

Figura 4. Classe Controladora das Classes de Projeto.

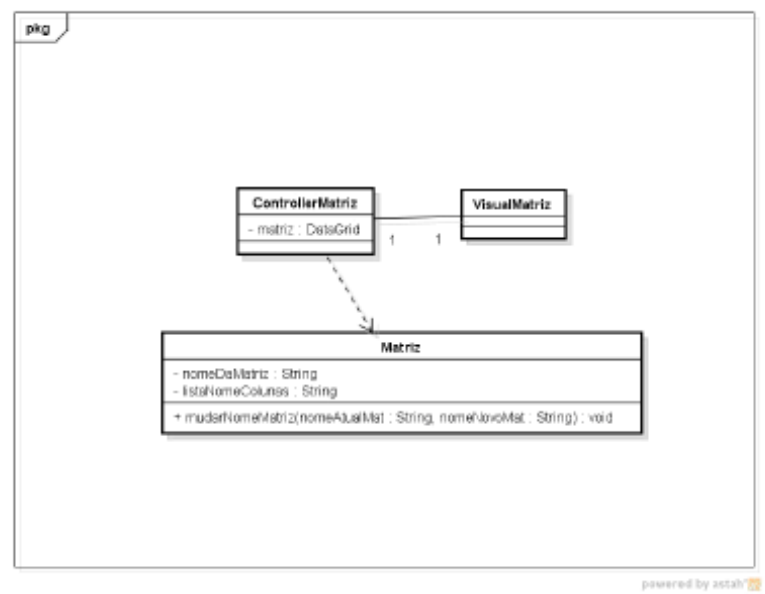

Figura 5. Classe Controladora das Classes de Matrizes.

Finalizado o processo de modelagem e documentação da ferramenta, deu-se início ao seu desenvolvimento. Foi escolhida a linguagem de programação Action Script por oferecer um melhor suporte para interface gráfica. As Figuras 6 e 7 mostram algumas telas da ferramenta.

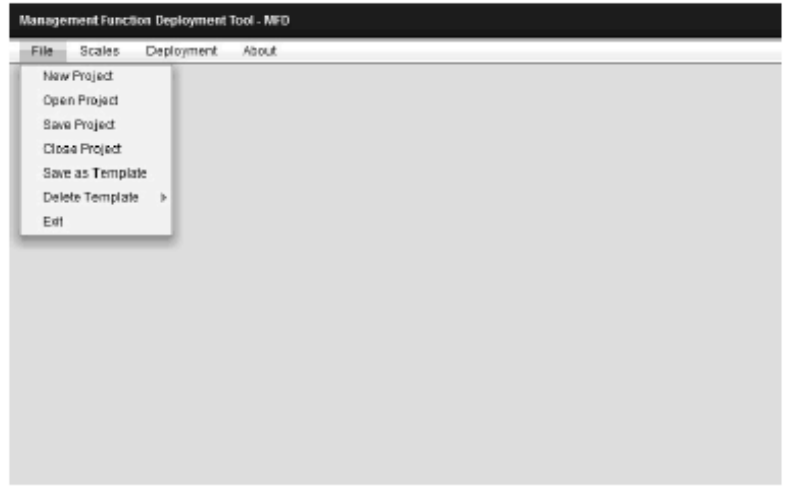

Figura 6. Tela Principal da Ferramenta.

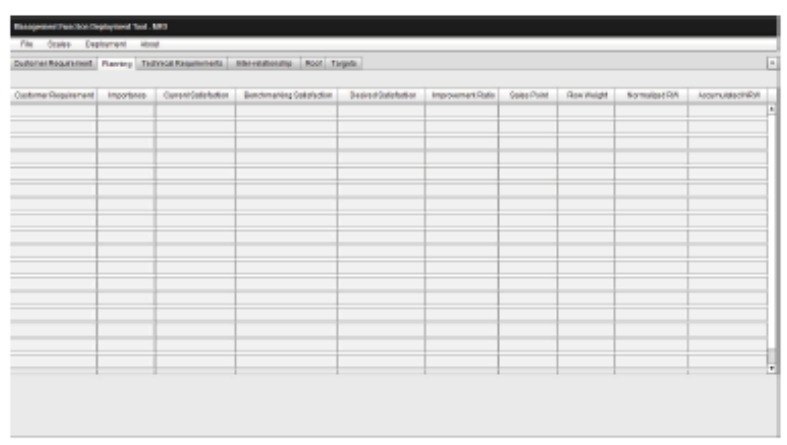

Figura 7. Tela da Matriz de Planejamento da House of Management (HoM).

\section{Conclusões}

Pode-se afirmar que o projeto de pesquisa conseguiu responder as perguntas de pesquisa propostas (P1 e P2), como também alcançar os seus objetivos através da realização da revisão sistemática e do desenvolvimento de uma versão inicial de uma ferramenta gratuita de apoio ao uso de técnicas baseadas no QFD.

A partir deste projeto de pesquisa, foi possível aprender diversos conceitos da área de Engenharia de Software dos quais destacam-se:

1 Conceitos básicos da área de Qualidade de Software;

2 Como elaborar e conduzir uma Revisão Sistemática de Literatura;

3 Como elicitar e documentar requisitos de software;

4 Como elaborar Diagramas de Caso de Uso e Diagramas de Classe;

5 Como desenvolver aplicações web utilizando a linguagem Action Script. 
Como principais trabalhos futuros, deseja-se (i) a conclusão da implementação da ferramenta e (ii) a sua avaliação prática através de um experimento controlado, conforme proposto inicialmente no projeto.

\section{Referências}

[1] BEECHAN, S. el al. Motivation in Software Engineering: A systematic literature review. Information and Software Technology: Elsevier, v. 50, n. 860-878, 2007.

[2] CARVAlHO, G. H. P.; SILVA, F. Q. B.; FRANÇA,A. C. C. Management Function Deployment: um Método para o Alinhamento Estratégico da Melhoria dos Processos de Gerenciamento de Projetos de Software. In: Proceedings do IX Simpósio Brasileiro de Qualidade de Software. Belém, PA, Brasil: SBQS, 2010, p. 135149.

[3] CARVAlHO, G. H. P.; SILVA, F. Q. B. Uma Proposta para o Alinhamento Estratégico da Melhoria dos Processos de Incubação Presentes no Modelo CERNE. In: Proceedings do XX Seminário Nacional de Parques Tecnológicos e Incubadoras de Empresas. Campo Grande, MS, Brasil: ANPROTEC, 2010.

[4] COHEN, L. Quality Function Deployment: How to Make QFD Work for You. Harrisonburg, VA, USA: Addison-Wesley Longman, 1995.

[5] CRAWFORD, J. K. Project Management Maturity Model. 2nd. ed. Pennsylvania, PA, USA: Auerbach Publications, 2006

[6] EASTERBROOK, S. 2007. Empirical research methods for software engineering. In Proceedings of the Twenty-Second IEEE/ACM international Conference on Automated Software Engineering. Atlanta, Georgia, USA: ASE, 2007.

[7] KERZNER, H. Using the Project Management Maturity Model: Strategic Planning for Project Management. 2nd. ed. New Jersey, NJ, USA: Wiley, 2005.

[8] KITCHENHAM, B. 2007. Guidelines for performing Systematic Literature Reviews in Software Engineering Version 2.3

[9] OGC. PRINCE2 Maturity Model. UK: Office of Government Commerce, 2006.
[10] OGC. Portfolio, Programme and Project Management Maturity Model. 2nd. ed. UK: Office of Government Commerce, 2008.

[11] PMI. Organizational Project Management MaturityModel (OPM3) - Knowledge Foundation (2nd Edition). 2nd. ed. Pennsylvania, PA, USA: ProjectManagement Institute, 2008.

[12] PRADO, D. Maturidade em Gerenciamento de Projetos. Minas Gerais, MG, Brasil: INDG Tecnologia e Serviços, 2008.

[13] SRIVANNABOON, S. Linking Project Management with Business Strategy. In: PMI Global Congress (North America). Washington, WA, USA: Project Management Institute, 2006.

[14] WOHLIN, C.; RUNESON, P; HÖST, M. Experimentation in Software Engineering: $\mathrm{Na}$ Introduction. Springer: 1999. 